\title{
Review
}

Simona Ferraro* and Mauro Panteghini

\section{Making new biomarkers a reality: the case of serum human epididymis protein 4}

https://doi.org/10.1515/cclm-2018-1111

Received October 15, 2018; accepted October 31, 2018; previously published online December 4, 2018

\begin{abstract}
Background: Measurement of human epididymis protein 4 (HE4) in serum has recently been proposed for clinical use in the framework of ovarian cancer (OvCa). We sought to retrace the translational phase and the clinical implementation steps boosting HE4's clinical value and discuss the effects of its introduction on the diagnostic and management pathways.
\end{abstract}

Methods: Meta-analyses of running evidence have preliminarily suggested that HE4 may overcome carbohydrate antigen 125 (CA125) in identifying OvCa, showing however several gaps that need to be considered, i.e. definition of biomarker diagnostic performance in the early detection of OvCa, added diagnostic value, biological and lifestyle factors of variation, and optimal interpretative criteria. Investigation of the influencing factors has shown that renal impairment represents a major limitation for HE4's diagnostic power. On the other hand, the demonstration of the substantial equivalence of results obtained by commercially available assays allows recommending harmonized thresholds for diagnostic purpose, even if the study of HE4's biological variation has clarified that the longitudinal interpretation of the biomarker changes according to the reference change value could be more appropriate. Summary: We used HE4 as an example for describing the long and bumpy road for making a new biomarker a reality, and the issues that should be checked and the information that should be provided in moving a novel biomarker from its discovery to an effective clinical adoption.

Keywords: diagnosis; immunoassay; ovarian cancer; prognosis; traceability.

*Corresponding author: Simona Ferraro, UOC Patologia Clinica, Ospedale "Luigi Sacco", Via GB Grassi 74, 20157 Milano, Italy; and Department of Biomedical and Clinical Sciences “Luigi Sacco", University of Milan, Milan, Italy, E-mail: simona.ferraro@asst-fbf-sacco.it

Mauro Panteghini: Department of Biomedical and Clinical Sciences “Luigi Sacco", University of Milan, Milan, Italy

\section{Introduction}

Although there is an overwhelming interest in biomarker research, in recent years scientific investigation has yielded relatively few new indicators worthy of clinical use [1]. Serum human epididymis protein 4 (HE4) is one of those biomarkers recently included in the narrow pipeline of clinical testing [2]. Since 2008 (the year of US Food and Drug Administration test clearance), we have observed a steep increase of articles about the diagnostic performance of HE4 in the ovarian cancer (OvCa) framework. HE4 represents a good example of candidate biomarker emerging from gene expression analysis and then translated into clinical practice.

The delivery of a tumor biomarker for clinical use is stepwise including discovery, translational and clinical phases. In the first phase, the marker is identified in the neoplastic tissue, generally by gene expression profiling studies [3]. The further analysis of protein expression in neoplasms first provides the opportunity for its application in histopathologic diagnosis [4]. The translational phase encompasses the comparison of gene expression in pathologic and normal tissues, in responders vs. non-responders to standardized chemotherapeutic protocols, and the correlation with the disease stage. The assay development and the further retrospective external validation allow gaining marketing approval and moving forward on the clinical introduction. During this phase, prospective evaluations should be planned to establish the applicability of the test in the population at risk, the capability of biomarker values to predict the progression of the disease and the individual response to treatment, as well as the association with survival. Finally, the effectiveness of the biomarker and the actual diagnostic contribution added to other clinical tools should be proved by estimating if and how its introduction has changed the morbidity and mortality trends.

Summarizing the concepts, the introduction of a new biomarker should answer four key questions: (1) Is there an unmet medical need? (2) Is the assay performance suitable? (3) Does the biomarker offer added clinical value? (4) Is the biomarker cost-effective? In this paper, we retrace the translational and clinical phases boosting HE4's 
implementation in the OvCa framework, by discussing how the knowledge about the biomarker characteristics may answer these questions and moves it to an effective clinical adoption potentially influencing patient outcome.

\section{Early diagnosis of OvCa: an unmet medical need}

The worldwide incidence of OvCa amounts to 190,000 new cases and the mortality to 114,000 deaths annually [5]. In Europe and United States, OvCa is the fourth most frequent cause of cancer death in women and the epithelial OvCa is the commonest cause of gynecological cancerassociated death. Often asymptomatic at early stages, OvCa is considered a "silent killer". Therapeutic intervention for a cure is possible in the early stage of the disease and this explains the direct relation between early diagnosis and survival [5]. The 5-year survival rate of $85 \%-90 \%$ at the International Federation of Gynecology and Obstetrics (FIGO) stage I disease (i.e. disease limited to ovaries only) decreases to $<20 \%$ at FIGO stage III (i.e. disease extending to the abdomen and/or lymph nodes) and IV (i.e. distant metastases) [6]. However, $75 \%$ of patients are diagnosed in stage II (i.e. disease extending to the pelvis) to IV for which the standard of care remains surgery and adjuvant platinum-based cytotoxic chemotherapy. Consequently, only a migration from late III/IV to early I/II stages would influence overall and disease-free survivals [5].

OvCa typically presents in post-menopausal women, most frequently between 55 and 64 years, and the highgrade serous represents the most common histological subtype of epithelial OvCa. However, the heterogeneous histopathology, the complexity of characterizing the resected tumor, the difference in incidence among subtypes (high-grade serous, $40 \%$; endometrioid, $25 \%$; clear cell, 26.4\%; mucinous, 6.2\%; low-grade serous, $2.4 \%$ ), directly influencing the biomarker expression and patient survival, have forced changing the notion of OvCa diagnosis from one to several distinct diseases and to target the treatment accordingly [7].

In general, the early diagnosis of malignancy in the population at risk is challenging, because the very low OvCa incidence ( $<40$ per 100,000 women) compels setting as desirable, for a diagnostic test, a specificity of $>99.5 \%$ to finally achieve a positive predictive value (PPV) of $10 \%$ [8]. This PPV, implying nine false positive test results for each true positive detecting OvCa, is the goal that currently gynecological oncologists consider as cost-effective for a diagnostic test [9]. In selecting the population at risk to be tested, one should take into account that $90 \%$ of OvCa are sporadic and diagnosed in post-menopausal women $>50$ years of age, and the incidence is higher when a pelvic mass is present, whereas less than $10 \%$ of OvCa are hereditary in women with a family history and BReast CAncer gene (BRCA) 1 and 2 mutations [5]. We should further consider that only $5 \%-10 \%$ of all women will be diagnosed with a pelvic mass, using current diagnostic tools, which include measurement of carbohydrate antigen 125 (CA125) in serum, imaging, pelvic exam, genetic analysis and family history. Running guidelines recommend that all patients with pelvic mass have surgery, but there is no indication about what type of surgeon and where the surgery should be performed [10]. The missing referral to gynecological oncology surgeons can result in a suboptimal management of OvCa (e.g. partial removal of cancer tissue, missing histopathological diagnosis and FIGO stage assessment) with a negative impact on patient survival $[5,6,11]$.

\section{Biomarkers in OvCa}

\section{Carbohydrate antigen 125}

CA125 is a glycoprotein belonging to the family of mucins (MW $>200 \mathrm{kDa}$ ), mainly expressed in tissues of Müllerian origin, designated as the elective biomarker in serous and endometrioid $\mathrm{OvCa}$ [12]. It is overexpressed in $80 \%$ of OvCa patients, but in only less than $50 \%$ of FIGO stage I disease, and in $\sim 1 \%$ of healthy people. As the measurement of CA125 in serum represents the biochemical "golden standard" in OvCa, the performance of new biomarkers has to be compared to it. However, a CA125 increase may occur in several benign diseases of gynecological origin or not (e.g., endometriosis, acute salpingitis, liver cirrhosis, heart failure), other malignancies, and miscellaneous conditions, like menstruation and pregnancy [13]. Thus, for clinical use CA125 values should be combined with imaging scores to increase specificity and PPV.

The 2011 National Institute for Health and Clinical Excellence (NICE) guidance for OvCa was meant to improve its early recognition, recommending CA125 measurement in primary care in symptomatic women presenting to general practitioners (GPs) [14]. A CA125 value above the threshold of $35 \mathrm{kU} / \mathrm{L}$ should be followed by an ultrasound scan (US) of the abdomen and pelvis in order to decide if there is a need to urgently refer or not the symptomatic woman to the specialist. Considering that the actual diagnostic sensitivity and specificity of CA125 amount to less than $80 \%$ [15] and the disease prevalence in women presenting with symptoms to primary care is $0.23 \%$, the estimated PPV of CA125 
results to be $<1 \%$, far from $10 \%$ value expected by oncologists $[8,9]$. The Prostate, Lung, Colorectal and Ovarian Cancer Screening Randomized Clinical Trial, enrolling 78,216 women (median follow-up of 12.4 years), showed that screening by CA125 and transvaginal US was unable to provide diagnostic anticipation when compared with usual care [16]. Furthermore, the consistent rate of false positive results was associated with an increase of invasive medical procedures, serious complications and related costs. Uncomfortable conclusions were also reached by auditing the NICE guideline application and reception. In the majority of cases, a dramatic increase of CA125 requests with inappropriate test ordering was the only recorded outcome [17-22]. Only one UK hospital reported that, in their experience, the majority of patients with CA125 $>35 \mathrm{kU} / \mathrm{L}$ were managed appropriately and the related PPV resulted in 20 -fold higher than expected [23]. For sure, further careful audit is required for actually assessing the reception of the NICE guidelines in healthcare practice and their impact in terms of early referral of women with OvCa to gynecologist oncologists and improvement of clinical outcome.

\section{Human epididymis protein 4}

First identified in the epithelium of distal epididymis, HE4 was found to be frequently overexpressed in OvCa and, to a lesser extent, in endometrioid and lung cancer [4]. HE4 is a member of the whey acidic proteins (WAP)gene family of protease inhibitors, characterized by an $\sim 50$ amino acid sequence with eight highly conserved cysteine residues forming four disulfide bridges [24]. It contains two WAP four-disulphide core (WFDC) domains. The native HE4 form is an $\mathrm{N}$-glycosylated protein of 20-25 kDa, synthesized with a signal peptide and two WFDC domains (N-WFDC, C-WFDC) [24]. Expression of HE4 in normal tissues occurs in the glandular epithelium of the female genital tract, except for ovarian surface epithelium, in epididymis and vas deferens, respiratory epithelium, distal renal tubules and salivary glands [4]. The molecular mechanism of action and the biological function of HE4 are still under investigation. HE4 promoted migration and adhesion of OvCa cells and the HE4 gene knockdown resulted in tumor growth inhibition [25]. HE4 also seemed to have a role in tumor progression: its overexpression in endometrial cancer cell lines induced cancer cell proliferation [26]. LeBleu et al. showed that HE4 acts as a protease inhibitor by decreasing the activity of serine proteases Prss35 and Prss23, which degrade the type I collagen that accumulates in kidney fibrosis [27]. Finally, HE4 may have a role in maintaining the innate immunity of the oral cavity and respiratory tract [28]. In cancer tissues other than ovarian, HE4 expression has been shown in lung adenocarcinomas and endometrial cancer, and, less often, in breast, gastrointestinal and urinary tract tumors [4, 29]. In OvCa, HE4 is strongly expressed in serous and poorly differentiated subtypes, in $50 \%-70 \%$ of endometrioid and clear cell tumors, but not in the mucinous histological type [4, 30].

Considering that HE4 expression in tumor tissues is basically restricted and highly up-regulated in OvCa and the protein is secreted in the bloodstream, the preconditions suggesting that this serological biomarker would be useful in the OvCa framework were fulfilled. Therefore, since 2009 clinical studies evaluating HE4 as biomarker for OvCa started to appear in the literature. In 2013, we metaanalyzed, after a systematic review, 16 of those studies comparing the diagnostic accuracy of CA125 and HE4 and aimed to assess the diagnostic contribution of the new marker [15]. Overall, HE4 showed it possessed the same sensitivity (79\%) but had a significantly higher specificity (93\%, confidence interval [CI]: 92-94) than CA125 (78\%, CI: 76-80). The higher specificity of HE4 vs. CA125 may be related, at least partly, to a lower rate of marker increases in benign gynecological diseases (Table 1) [31].

However, some important unanswered questions were raised in commenting on meta-analysis results [15]. Firstly, although the combined measurement of HE4 and CA125 was the widely recommended application of the new marker, the evaluation of the diagnostic performance of the combined measurements was considered only in a few studies. Secondly, very few articles evaluated biomarker performance in the detection of OvCa at an early stage (FIGO I/II). Lastly, although statistics assign the highest baseline risk index for OvCa to post-menopausal women, there was a scarce focus on menopausal status. In a following paper, we further listed what information was still needed before deciding for the clinical introduction of HE4 [8]:

- to verify the status of harmonization of commercially available assays and the comparability of HE4 results as well as to assess the possible effects on patients' clinical classification;

- to identify biological and lifestyle factors influencing HE4 concentrations in serum;

- to define the most adequate interpretation strategy (i.e. age-related reference limits, fixed decision limits or reference change value [RCV]);

- to decide if CA125 should be replaced or whether HE4 should be offered in combination with it;

- to know if HE4 measurement impacts better on patient outcome and leads to more cost-effective management compared to CA125. 
Table 1: Rates of HE4 vs. CA125 increase in benign gynecological diseases.

\begin{tabular}{|c|c|c|c|c|}
\hline \multirow[t]{2}{*}{ Benign disease } & \multicolumn{2}{|c|}{ Premenopausal } & \multicolumn{2}{|c|}{ Post-menopausal } \\
\hline & HE4 & CA125 & HE4 & CA125 \\
\hline Ovarian cysts & $6 \%$ & $15 \%$ & $13 \%$ & $13 \%$ \\
\hline Sex cord stromal tumors & $20 \%$ & $60 \%$ & $25 \%$ & $50 \%$ \\
\hline Cystadenoma, adenofibroma, cystadenofibroma & $20 \%$ & $15 \%$ & $19 \%$ & $22 \%$ \\
\hline Endometriosis/endometrioma & $3 \%$ & $72 \%$ & $6 \%$ & $18 \%$ \\
\hline Abscess/hydrosalpinx/pelvis inflammatory disease & $13 \%$ & $40 \%$ & $13 \%$ & $33 \%$ \\
\hline Fibroid (leiomyomas) & $9 \%$ & $41 \%$ & $7 \%$ & $7 \%$ \\
\hline Benign, other (normal ovaries) & $7 \%$ & $29 \%$ & $4 \%$ & $17 \%$ \\
\hline
\end{tabular}

Adapted from Ref. [31]. In italics significant differences $(p<0.05)$ between HE4 and CA125 rates of increase.

\section{Suitability of available HE4 assays}

Running immunoassays for serum HE4 measurement are reported to differ in monoclonal antibodies (MoAb) employed and in principles (Figure 1) [32]. Assay differences in antibody selectivity, signal detection and method design may influence biomarker results and affect clinical performance [33]. In general, scarce data on method comparability in HE4 measurement were available, even if some automated assays report in their package inserts the same decisional limits [34]. Only a head-to-head method comparison showing result equivalence could enable using harmonized cut-off values when measuring HE4 by different assays.

All automated immunoassays that are currently commercially available declare being traceable to manual

\begin{tabular}{|l|c|c|c|c|}
\hline Platform & $\begin{array}{c}\text { Manual } \\
\text { Fujirebio }\end{array}$ & $\begin{array}{c}\text { Fujirebio } \\
\text { Diagnostics }\end{array}$ & $\begin{array}{c}\text { Roche } \\
\text { Diagnostics }\end{array}$ & $\begin{array}{c}\text { Abbott } \\
\text { Diagnostics }\end{array}$ \\
\hline Assay principle & - & $\begin{array}{c}\text { Lumipulse } \\
\text { G1200 }\end{array}$ & $\begin{array}{c}\text { Modular, } \\
\text { Elecsys 2010; } \\
\text { Cobas e411, } \\
601,602,801\end{array}$ & $\begin{array}{c}\text { Architect } \\
\text { series }\end{array}$ \\
\hline MoAb & $\begin{array}{c}\text { Two-step non } \\
\text { competitive } \\
\text { sandwich }\end{array}$ & $\begin{array}{c}\text { Two-step non } \\
\text { competitive } \\
\text { sandwich }\end{array}$ & $\begin{array}{c}\text { One-step } \\
\text { sandwich }\end{array}$ & $\begin{array}{c}\text { Two-step non } \\
\text { competitive } \\
\text { sandwich }\end{array}$ \\
\hline Principle & $2 \mathrm{H}$ \& 3D8 & $2 \mathrm{H} 5$ \& 12A2 & $2 \mathrm{H} 5$ \& 12A2 & $2 \mathrm{H} 5$ \& 3D8 \\
\hline $\begin{array}{l}\text { Manufacturer's suggested } \\
\text { cut-off (pmol/L) }\end{array}$ & EIA & CLEIA & ECLIA & CMIA \\
\hline
\end{tabular}

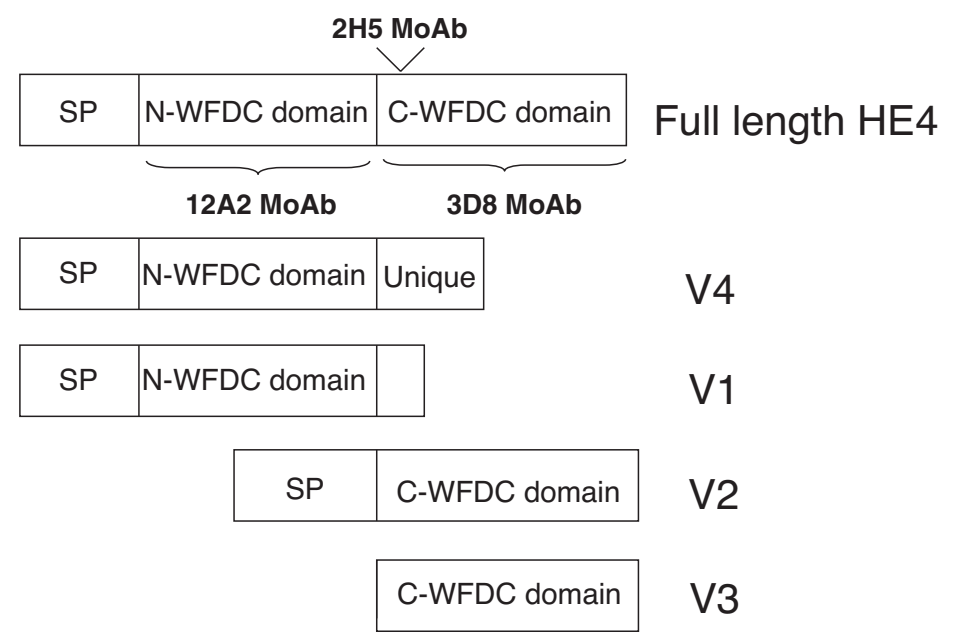

Figure 1: Characteristics of commercially available assays for HE4 determination and their selectivity for biomarker variants. preM, premenopausal women; postM, post-menopausal women; SP, signal peptide. 
EIA manufactured by Fujirebio Diagnostics (and in turn to the standard preparation employed for its calibration) used to provide the first evidence of the HE4 clinical value. In the metrological traceability purport, EIA should, therefore, be considered as the "higher order reference procedure" in the HE4 traceability chain [32]. The manual EIA was developed based on $2 \mathrm{H} 5 \mathrm{MoAb}$ as catching MoAbs and 3D8 MoAb as detecting MoAbs, respectively. EIA calibrator values are assigned against the in-house standard consisting of recombinant immunoglobulin (human Fc antibody fragment)-HE4 fusion protein. Abbott Diagnostics uses the same MoAbs of EIA, whereas Roche Diagnostics and the automated Fujirebio assay on the Lumipulse platform replaces 3D8 with 12A2 MoAb (Figure 1). According to the employed MoAbs, EIA and Architect assays would recognize all HE4 variants except V4, while Roche and Lumipulse assays would not be able to detect HE4 V2 and V3 variants, which however are usually not present in plasma. These HE4 variants appear to exhibit variable expression in OvCa as well as in benign ovarian disease and in other non-neoplastic conditions [35].

Summarizing data obtained in an ad hoc study, we showed: (a) for the Architect assay, an optimal alignment against the EIA method; (b) for the Roche assay, a good agreement with EIA at HE4 concentrations $<250 \mathrm{pmol} / \mathrm{L}$, with a clinically significant underestimation of HE4 values at higher concentrations; (c) for Fujirebio Lumipulse, a suboptimal alignment due to a marked systematic overestimation of HE4 values, to be corrected by an appropriate assay recalibration [32]. Overall, our data showed that the Abbott and Roche assays exhibit a good comparability in the range of HE4 values around the previously recommended EIA cut-off of $140 \mathrm{pmol} / \mathrm{L}$. Consequently, a common threshold level may be adopted to rule in patients with suspected OvCa when these two assays are used. However, considering the significant variability of inter-method bias along the whole range of HE4 concentrations, individual monitoring with serial measurements for longitudinal evaluation of biomarker changes must be only performed using the same assay.

\section{Reference intervals for serum HE4}

For HE4 a wide range of decision thresholds has been reported in literature. No consensus is achieved in this framework and there are still net discrepancies between reference intervals recommended by various manufacturers in their package inserts, also for those assays employing the same antibodies (Figure 1).

\section{The individual biology of OvCa biomarkers}

The lack of studies investigating the biological variation of HE4 represented another major shortcoming for its effective clinical application. On the other hand, the few available studies evaluating CA125 biological variation suffered major limitations, enrolling individuals with potential interfering conditions (e.g., carrying other malignancies), collecting serial samples at a variable time distance, also during menstruation, or missing the partitioning of subjects for menopausal status [36-38]. Therefore, we performed a study to estimate the biological variation parameters of CA125 and HE4 in the same cohort of women, by adopting an accurately designed experimental protocol paying attention to menopausal status, preanalytical sources of variability and to statistical analysis of data [39]. For both markers, we found no differences in serum concentrations between pre- and post-menopausal women. For CA125, intra-individual variances were also not different between the two groups, while the within-subject biological CV for HE4 was approximately 2 times higher in pre- than in post-menopausal women $(12.1 \%$ vs. $6.5 \%, p<0.001)$. The index of individuality for both markers was $<0.6$, meaning that population-based reference limits may have little use in the interpretation of marker results in a particular individual [40]. According to biological variation data, monitoring longitudinal changes in individual serum concentrations of OvCa biomarkers over time by RCV appears more appropriate than using single threshold rules [41]. Employing the Roche assay (or assays with similar imprecision), an RCV for HE4 of approximately $35 \%$ for pre- and $20 \%$ for post-menopausal women, respectively, was estimated [39]. These data highlighted that pre- and post-menopausal women who have undergone HE4 detection have to be managed differently by resorting to an appropriate RCV value, according to differences in biological variation due to the hormonal status. In agreement with a better effectiveness of longitudinal biomarker interpretation, Drescher et al. [42] demonstrated that, in using CA125 for detecting OvCa, an algorithm that incorporates change over time in individual biomarker concentrations might identify the malignancy on average 10 months earlier than a single threshold rule in a meaningful proportion of cases. 


\section{Factors of variation of serum HE4}

\section{Age and menopause}

Various biological and behavioral factors have been suggested to influence HE4 concentrations in serum, thus potentially limiting its diagnostic capability [43]. Age, renal function, parity, smoking and caffeine consumption appeared to influence HE4 levels. Women aged $>55$ years display higher HE4 concentrations than younger individuals [44]. The relationship between age and marker concentrations is non-linear and by assuming 20 years of age as baseline, HE 4 concentrations rise by $2 \%$ at 30 years, $9 \%$ at 40 years, $20 \%$ at 50 years, $37 \%$ at 60 years, $63 \%$ at 70 years and $101 \%$ at 80 years of age [45]. Although different decisional levels have been proposed to interpret HE4 results in pre- and post-menopausal women, we were unable to find definitive evidence supporting changes of HE4 concentrations in relation to menopausal status [39]. Menopause per se may not be considered an influencing condition: in some studies, pre-menopausal women appeared to exhibit HE4 concentrations significantly lower than those in post-menopausal women, but this difference became not significant when restricting the comparison to premenopausal and post-menopausal women $<60$ years [46-49]. According to results by Yang et al. [47], women aged $>60$ years are the only age group with HE4 concentrations significantly higher. Undoubtedly, menopause modulates the biological behavior of HE4 concentrations in serum, which relevantly change from pre- to post-menopausal period by assuming a specific pattern of marker release modulated by the different hormonal status [39]. Controversial data are available on the effect of menstrual cycle on HE4. Although Anastasi et al. [50] first reported HE4 concentrations lower in follicular vs. ovulatory phase in women $<35$-years-old, more recently Hallamaa et al. [51] did not find any difference in serum HE4 concentrations in different phases of menstrual cycle.

\section{Smoking and body mass index (BMI)}

HE4 concentrations in serum were increased by $20 \%-30 \%$ in smokers when compared with non-smokers [44, 45, 52]. The possibility that HE4 release is enhanced by BMI lowering might have a relevant impact in those patients with OvCa treated with chemotherapy, undergoing weight loss and being monitored by HE4 assaying during the followup [53]. We performed an ad hoc study, properly projected to exclude or control other confounding factors of HE4 variation [54]. We enrolled 103 women without current or history of ovarian disease or other gastrointestinal/ gynecological benign or malignant diseases, not smokers, aged $\leq 55$ years and with serum creatinine concentrations $\leq 0.96 \mathrm{mg} / \mathrm{dL}$ (i.e. the upper reference limit for adult women in our institution), and a BMI ranging from 19 to $57 \mathrm{~kg} / \mathrm{m}^{2}$. The overall case series was partitioned into three groups according to BMI values. While age, renal function and use of oral contraceptives significantly influenced HE4 concentrations in serum, changes in BMI did not affect them [54].

\section{Renal function}

An unquestionable evidence is that the main factor potentially hindering the clinical use of HE4 for OvCa detection and management is the presence of a renal impairment [5557]. In 181 women, including subjects with physiological renal and ovarian function and patients at different stages of chronic kidney disease (CKD), with no ovarian and lung cancer, Nagy et al. reported a significant increase of HE4 concentrations proportional to the decrease in glomerular filtration rate (GFR), while CA125 was slightly increased only in CKD at the terminal stage [55]. This posed the question if a GFR impairment could represent the "Achilles' heel" of HE4 by hampering its specificity for OvCa detection, and how to protect the diagnostic potential of this test [57]. This issue is not easy to solve as a considerable expression of the gene encoding for HE4 has been characterized in renal tissue and studies have shown an increase of biomarker concentrations in patients with any grade of kidney injury as well as with fibrotic kidney $[4,58,59]$. Assessing the effect of GFR changes on serum HE4 concentrations in subjects with physiological or mildly decreased glomerular function sounds relevant for interpreting biomarker concentrations in neoplastic patients treated with chemotherapy agents, which represent a major risk factor for the development of renal impairment as kidneys often metabolize and excrete them [60]. To this purpose, we enrolled a population of 101 women aged $<56$ years with a GFR ranging from 60 to $120 \mathrm{~mL} / \mathrm{min} / 1.73 \mathrm{~m}^{2}$, free of any disease and biological and lifestyle factors known to influence serum HE4 concentrations [61]. Regression models showed that the serum cystatin $\mathrm{C}$ measurement was the most sensitive indicator to catch HE4 increases due to a mild decrease in renal function (adjusted $r^{2}, 0.38 ; p=0.00003$ ). By assuming a cystatin $\mathrm{C}$ concentration of $0.80 \mathrm{mg} / \mathrm{L}$ as baseline, a $0.10 \mathrm{mg} / \mathrm{L}$ elevation of this protein in serum implies an average increase of $9.2 \mathrm{pmol} / \mathrm{L}$ of HE4 [61]. Accordingly, we suggested in the OvCa follow-up, the simultaneous monitoring of HE4 and cystatin C concentrations in serum may 
allow better discrimination of a clinically relevant HE4 variation due to the progression of the disease from the one related to an impairment of renal function [61].

\section{Introducing HE4: which added clinical value?}

Looking at biological variability data and at other biological and lifestyle factors potentially influencing the two biomarkers [39, 43], it is likely that CA125 and HE4 may have a different individual behavior that could make it difficult to draw robust inference on their predictive clinical value. OvCa histological subtypes represent a further factor to be considered when interpreting CA125/HE4 results. Theoretically, HE4 should outperform CA125 in serous and endometrioid subtypes. On the contrary, for mucinous and clear cell tumors CA125 should be preferentially tested [13].

\section{Diagnostic performance}

In the mentioned meta-analysis [15], HE4 measurement seemed to be superior to CA-125 in terms of diagnostic performance for the identification of $\mathrm{OvCa}$ in women with suspected gynecological disease. The risk for OvCa was significantly increased for patients with HE4 positive results (odds ratio, 37.2 for HE4 vs. 15.4 for CA125, respectively) and the calculation of positive likelihood ratio confirmed that HE4 outperforms CA125 in identifying OvCa (13.0 vs. 4.2) [15]. Another group reported similar results [62]. According to this preliminary evidence, it was suggested to replace CA125 with HE4 for OvCa detection. However, before replacing the CA125 information, a more robust estimate of HE4 diagnostic performance is needed. In particular, well-designed prospective clinical trials are required to reinforce this preliminary evidence.

Since HE4 introduction, some manufacturers recommended the Risk of Ovarian Malignancy Algorithm (ROMA), which utilizes the HE4/CA125 combination along with the menopausal status, to triage patients with pelvic mass. Although ROMA ${ }^{\mathrm{TM}}$ has received clearance from the US Food and Drug Administration, there have been concerns raised about its clinical validation leading to controversial results about its diagnostic accuracy when compared to CA125 and HE4 alone [63-65]. A recent systematic review observed that although ROMA may increase the diagnostic sensitivity when compared with markers alone, the specificity of ROMA was significantly lower than that of HE4 alone (82\%, CI: $79-85$, vs. $87 \%$, CI: 85-89, respectively) [66].

\section{Monitoring response to therapy}

The measurement of serum CA125 during chemotherapy is used to evaluate the response to the treatment, in addition to imaging and clinical assessment. The National Comprehensive Cancer Center Guidelines recommend follow-up visits every 2-4 months for the first 2 years followed by 6-month intervals for the next 3 years [67]. At each visit, a physical examination and CA125 measurement are always recommended, whereas imaging examination should be done only if clinically indicated.

In a recently published prospective case series of 43 OvCa patients undergoing tumor monitoring, in whom paired serial measurement of CA125 and HE4 were obtained, the rate of significant biomarker decrease after therapy was higher for CA125 than for HE4 (28.4\% vs. $14.6 \%, p=0.006$ ) [68]. From this analysis, CA125 seemed to outperform HE4 as biomarker to monitor the response to successful chemotherapy in OvCa patients, even in situations of preserved renal function. Other authors have recently confirmed these data [69]. Once again, this initial evidence should be confirmed by additional comparative studies with larger sample sizes in order to provide a definitive conclusion.

\section{Surveillance of OvCa recurrence}

Despite the achievement of a complete clinical response, OvCa recurs in $25 \%$ of patients with an early-stage disease and in $>80 \%$ of patients in advanced stages [70]. The follow-up with biomarkers may permit the early detection of OvCa recurrence and delay the onset of cancer-related symptoms (e.g., ascites, bowel obstruction), improving the quality of life of patients. Furthermore, biomarker monitoring may aid in selecting patients who may benefit from secondary cytoreductive surgery. Finally, considering the availability of new treatment options, the measurement of serum biomarkers may represent a surrogate outcome allowing an early change of chemotherapeutic strategy in light of the evidence of rising concentrations. The common belief that an earlier treatment of tumor relapse based on raised CA125 concentrations alone can result in better outcome has been, however, challenged by the results from the MRC OV05/EORTC 55955 trial [71].

Considering the detection of OvCa recurrence, some authors have suggested the superiority of HE4 over CA125 measurements [72, 73]. As we have previously mentioned, both biomarkers suffer for high individuality: thereby, monitoring serial individual changes over time can be more appropriate than using fixed threshold rules. In a 
Table 2: Cost-effectiveness of different triage strategies for adnexal masses in premenopausal and post-menopausal women.

\begin{tabular}{|c|c|c|c|c|c|}
\hline Strategy & Mean cost $^{\mathrm{a}}, €$ & Cases detected & Cost per case detected, $€$ & $\begin{array}{r}\text { Cases diagnosed } \\
\text { correctly }\end{array}$ & $\begin{array}{r}\text { Cost per patient diagnosed } \\
\text { correctly, } €\end{array}$ \\
\hline \multicolumn{6}{|c|}{ Premenopausal women } \\
\hline TVS & 36.41 & $8 \%$ & 447.10 & $95 \%$ & 38.10 \\
\hline TVS + CA125 & 49.41 & $8 \%$ & 606.70 & $66 \%$ & 74.80 \\
\hline TVS + HE4 & 57.12 & $8 \%$ & 701.40 & $87 \%$ & 65.40 \\
\hline \multicolumn{6}{|c|}{ Post-menopausal women } \\
\hline TVS & 39.52 & $30 \%$ & 131.70 & $83 \%$ & 47.60 \\
\hline TVS + CA 125 & 52.52 & $34 \%$ & 153.90 & $82 \%$ & 64.20 \\
\hline TVS + HE4 & 60.23 & $32 \%$ & 186.20 & $85 \%$ & 71.10 \\
\hline
\end{tabular}

TVS, transvaginal sonography with subjective assessment by an experienced ultrasound operator when TVS results were inconclusive. aThe slightly higher TVS costs in post-menopausal women may be related to an increased use of a second TVS performed by an expert consultant for the re-evaluation of mass with conflicting features. The use of a level 3 evaluation (as defined by the European Federation of Societies for Ultrasound in Medicine and Biology) in addition to the preliminary simple rules may explain the increased costs. On the contrary, marker costs are unchanged in the two groups (always $€ 3.00$ for CA125 and €20.71 for HE4). Data from Ref. [74].

recent study [68], we investigated the two approaches for defining a biochemical relapse and their agreement with the clinical evidence of disease progression: (a) an absolute increase of CA125/HE4 concentrations in serum above the established decision limit, and (b) a percentage variation in CA125/HE4 concentrations in serum exceeding respective RCVs. We observed that the use of CA125 according to the cut-off rule (i.e. an increase above $35 \mathrm{kU} / \mathrm{L}$ ) appeared to completely fit the clinical evaluation of relapse, while HE4 showed a 4.3\% rate of false positive results [68]. The use of the RCV rule increased the rate of false positives for both markers (8.3\% for CA125 and 13.3\% for HE4, respectively). In 11 patients undergoing various chemotherapy protocols, we detected 21 isolated CA125 elevations, all associated to a disease progression assessed by computed tomography scan and positron emission tomography. For HE4, we detected seven isolated increases according to the RCV in the serum of six patients undergoing various chemotherapy regimens. Five of those were recorded simultaneously to renal impairment occurring after chemotherapy [68]. Therefore, our study data was a relevant warning of the similarity of CA125 and HE4 behavior in OvCa follow-up. This happens predominantly because of renal impairment or different response to the treatment by the two biomarkers. Overall, CA125 appeared the most reliable marker for disease monitoring, whereas HE4 contributes additional information only in a minority of cases.

\section{HE4 cost-effectiveness}

We were able to find only one paper dealing with the costeffectiveness of HE4 use in the initial triage of women with pelvic masses [74]. While the addition of CA125 to transvaginal US proved useful enough to triage those patients (but only in post-menopausal group), the study did not support the cost-effectiveness of HE4 use in replacing CA125 in the initial triage of adnexal masses (Table 2).

\section{Conclusions}

In this paper, we used HE4 as an example for describing the long and bumpy road for making a new biomarker a reality, and the issues that should be checked and the information that should be provided in moving a novel biomarker from its discovery to its effective clinical adoption $[75,76]$. From what we have discussed, it appears that further efforts are still needed to fill the existing gap between the basic science background and the assay development and validation, and the final clinical compliance of HE4. Considering that HE4 is a biomarker "competing" with CA125 for clinical use, the evidence of the frequent disagreement between the two markers is likely to complicate the patient triage, raising doubts on possible changes in treatment strategies and triggering further diagnostic tests. Unmet aspects require being approached with further ad hoc clinical trials [77]. As very often happens with new biomarkers, the audit of clinical outcomes (e.g. improved survival and cost-benefit ratio) represents the major challenge [78].

Author contributions: All the authors have accepted responsibility for the entire content of this submitted manuscript and approved submission.

Research funding: None declared. 
Employment or leadership: None declared.

Honorarium: None declared.

Competing interests: The funding organization(s) played no role in the study design; in the collection, analysis, and interpretation of data; in the writing of the report; or in the decision to submit the report for publication.

\section{References}

1. Ptolemy AS, Rifai N. What is a biomarker? Research investments and lack of clinical integration necessitate a review of biomarker terminology and validation schema. Scand J Clin Lab Invest 2010;70(Suppl 242):6-14.

2. Anderson NL. The clinical plasma proteome: a survey of clinical assays for proteins in plasma and serum. Clin Chem 2010;56:177-85.

3. Lu KH, Patterson AP, Wang L, Marquez RT, Atkinson EN, Baggerly $K A$, et al. Selection of potential markers for epithelial ovarian cancer with gene expression arrays and recursive descent partition analysis. Clin Cancer Res 2004;10:3291-300.

4. Galgano MT, Hampton GM, Frierson HF Jr. Comprehensive analysis of HE4 expression in normal and malignant human tissues. Mod Pathol 2006;19:847-53.

5. Jayson GC, Kohn EC, Kitchener HC, Ledermann JA. Ovarian cancer. Lancet 2014;384:1376-88.

6. Prat J, for the FIGO Committee on Gynecologic Oncology. Staging classification for cancer of the ovary, fallopian tube, and peritoneum. Int J Gynaecol Obstet 2014;124:1-5.

7. Köbel M, Kalloger SE, Boyd N, McKinney S, Mehl E, Palmer C, et al. Ovarian carcinoma subtypes are different diseases: implications for biomarker studies. PLoS Med 2008;5:e232.

8. Ferraro S, Panteghini M. Is serum human epididymis protein 4 ready for prime time? Ann Clin Biochem 2014;51:128-36.

9. Menon U, Jacobs IJ. Ovarian cancer screening in the general population: current status. Int J Gynecol Cancer 2001;11:3-6.

10. Graham L. ACOG releases guidelines on management of adnexal masses. Fam Physician 2008;77:1320-3.

11. Junor EJ, Hole DJ, McNulty L, Mason M, Young J. Specialist gynaecologists and survival outcome in ovarian cancer: a Scottish national study of 1866 patients. Br J Obstet Gynaecol 1999;106:1130-6.

12. Yin BW, Lloyd KO. Molecular cloning of the CA125 antigen: identification as a new mucin, MUC 16. J Biol Chem 2001;276:27371-5.

13. Nolen BM, Lokshin AE. Protein biomarkers of ovarian cancer: the forest and the trees. Future Oncol 2012;8:55-71.

14. National Collaborating Centre for Cancer (UK). National Institute for Health and Clinical Excellence (NICE): Guidance. Ovarian cancer: the recognition and initial management of ovarian cancer. Cardiff, UK: National Collaborating Centre for Cancer, 2011.

15. Ferraro S, Braga F, Lanzoni M, Boracchi P, Biganzoli EM, Panteghini M. Serum human epididymis protein 4 vs carbohydrate antigen 125 for ovarian cancer diagnosis: a systematic review. J Clin Pathol 2013;66:273-81.

16. Buys SS, Partridge E, Black A, Johnson CC, Lamerato L, Isaacs C, et al. PLCO project team. Effect of screening on ovarian cancer mortality: the prostate, lung, colorectal and ovarian (PLCO) cancer screening randomized controlled trial. J Am Med Assoc 2011;305:2295-303.

17. Porchetta C, Armstrong WE, Boa FG. A comparison of CA125 requests pre- and post-NICE Guideline CG122: recognition and initial management of ovarian cancer. Ann Clin Biochem 2012;49(Suppl 1):27.

18. Darwin LL. The use of CA125 following publication of NICE guidance for the recognition and initial management of ovarian cancer. Ann Clin Biochem 2012;49(Suppl 1):28.

19. Ghandhi I, Lee V, Madira W. Primary care adherence to NICE guidelines (CG122) on suspected ovarian cancer. Ann Clin Biochem 2012;49(Suppl 1):29.

20. Hassall LC, Al-Jubouri MA. An audit of CA125 requesting since the introduction of NICE guidelines CG122. Ann Clin Biochem 2012;49(Suppl 1):29.

21. Ford A, Marshall O, Crawford SM. UK guidance on diagnosis of ovarian cancer: its impact on services in an acute hospital. Eur J Cancer 2013;49(Suppl 2):S742.

22. Crawford SM, Evans C. Outcome of elevated CA125 values from primary care following implementation of ovarian cancer guidelines. Fam Pract 2018;35:199-202.

23. Garner $\mathrm{E}$, Thompson D. CA125 requesting in primary care: more effective than expected. Ann Clin Biochem 2012;49(Suppl 1):30.

24. Bouchard D, Morisset D, Bourbonnais Y, Tremblay GM. Proteins with whey-acidic-protein motifs and cancer. Lancet Oncol 2006;7:167-74.

25. Lu R, Sun X, Xiao R, Zhou L, Gao X, Guo L. Human epididymis protein 4 (HE4) plays a key role in ovarian cancer cell adhesion and motility. Biochem Biophys Res Commun 2012;419:274-80.

26. Li J, Chen H, Mariani A, Chen D, Klatt E, Podratz K, et al. HE4 (WFDC2) promotes tumor growth in endometrial cancer cell lines. Int J Mol Sci 2013;14:6026-43.

27. LeBleu VS, Teng Y, O'Connell JT, Charytan D, Müller GA, Müller CA, et al. Identification of human epididymis protein- 4 as a fibroblast-derived mediator of fibrosis. Nat Med 2013;19:227-31.

28. Bingle L, Cross SS, High AS, Wallace WA, Rassl D, Yuan G, et al. WFDC2 (HE4): a potential role in the innate immunity of the oral cavity and respiratory tract and the development of adenocarcinomas of the lung. Respir Res 2006;7:61.

29. Simmons AR, Baggerly K, Bast RC Jr. The emerging role of HE4 in the evaluation of epithelial ovarian and endometrial carcinomas. Oncology (Williston Park) 2013;27:548-56.

30. Kristjansdottir B, Levan K, Partheen K, Sundfeldt K. Diagnostic performance of the biomarkers HE4 and CA125 in type I and type II epithelial ovarian cancer. Gynecol Oncol 2013;131:52-8.

31. Moore RG, Miller MC, Steinhoff MM, Skates SJ, Lu KH, LambertMesserlian G, et al. Serum HE4 levels are less frequently elevated than CA125 in women with benign gynecologic disorders. Am J Obstet Gynecol 2012;206:351.e1-8.

32. Ferraro S, Borille S, Carnevale A, Frusciante E, Bassani N, Panteghini M. Verification of the harmonization of human epididymis protein 4 assays. Clin Chem Lab Med 2016;54:1635-43.

33. Madsen JS, Nybo M, Magid E, Hilden J, Hornung N, Larsen TB, et al. More studies on outcomes using biochemical diagnostic tests are needed: findings from the Danish Society of Clinical Biochemistry. Clin Chem 2008;54:1254-6.

34. Ruggeri G, Bandiera E, Zanotti L, Belloli S, Ravaggi A, Romani C, et al. HE4 and epithelial ovarian cancer: comparison and clinical evaluation of two immunoassays and a combinations algorithm. Clin Chim Acta 2011;412:1447-53. 
35. Jiang SW, Chen H, Dowdy S, Fu A, Attewell J, Kalogera E, et al. HE4 transcription and splice variant specific expression in endometrial cancer and correlation with patient survival. Int J Mol Sci 2013;14:22655-77.

36. Browning MC, McFarlane NP. Objective interpretation of results for tumour marker. J Nucl Med Allied Sci 1990;34(Suppl):89s-91.

37. Tuxen MK, Sölétormos G, Petersen PH, Schiǿler V, Dombernowsky P. Assessment of biological variation and analytical imprecision of CA 125, CEA, and TPA in relation to monitoring of ovarian cancer. Gynecol Oncol 1999;74:12-22.

38. Trapé J, Pérez de Olaguer J, Buxó J, López L. Biological variation of tumor markers and its application in the detection of disease progression in patients with non-small cell lung cancer. Clin Chem 2005;51:219-22.

39. Braga F, Ferraro S, Mozzi R, Panteghini M. The importance of individual biology in the clinical use of serum biomarkers for ovarian cancer. Clin Chem Lab Med 2014:52:1625-31.

40. Harris EK. Effects of intra- and inter-individual variation on the appropriate use of normal range. Clin Chem 1974;20:1535-42.

41. Braga F, Panteghini M. Generation of data on within-subject biological variation in laboratory medicine: An update. Crit Rev Clin Lab Sci 2016;53:313-25.

42. Drescher CW, Shah C, Thorpe J, O'Briant K, Anderson GL, Berg $C D$, et al. Longitudinal screening algorithm that incorporates change over time in CA125 levels identifies ovarian cancer earlier than a single-threshold rule. J Clin Oncol 2013;31:387-92.

43. Ferraro S, Schiumarini D, Panteghini M. Human epididymis protein 4: factors of variation. Clin Chim Acta 2014;438:171-7.

44. Urban N, Thorpe J, Karlan BY, McIntosh MW, Palomares MR, Daly $M B$, et al. Interpretation of single and serial measures of HE4 and CA-125 in asymptomatic women at high risk for ovarian cancer. Cancer Epidemiol Biomarkers Prev 2012;21:2087-94.

45. Bolstad N, Oijordsbakken M, Nustad K, Bjerner J. Human epididymis protein 4 reference limits and natural variation in a Nordic reference population. Tumour Biol 2012;33:141-8.

46. Escudero JM, Auge JM, Filella X, Torne A, Pahisa J, Molina R. Comparison of serum human epididymis protein 4 with cancer antigen 125 as a tumor marker in patients with malignant and nonmalignant diseases. Clin Chem 2011;57:1534-44.

47. Yang J, Sa M, Huang M, Yang J, Xiang Z, Liu B, et al. The reference intervals for HE4, CA125 and ROMA in healthy female with electrochemiluminescence immunoassay. Clin Biochem 2013;46:1705-8.

48. Moore RG, Miller MC, Eklund EE, Lu KH, Bast RC Jr, LambertMesserlian G. Serum levels of the ovarian cancer biomarker HE4 are decreased in pregnancy and increase with age. Am J Obstet Gynecol 2012;206:349.e1-7.

49. Park Y, Kim Y, Lee EY, Lee JH, Kim HS. Reference ranges for HE4 and CA125 in a large Asian population by automated assays and diagnostic performances for ovarian cancer. Int J Cancer 2012;130:1136-44.

50. Anastasi E, Granato T, Marchei GG, Viggiani V, Colaprisca B, Comploj S, et al. Ovarian tumor marker HE4 is differently expressed during the phase of menstrual cycle in healthy young women. Tumour Biol 2010;31:411-5.

51. Hallamaa M, Suvitie P, Huhtinen K, Matomäki J, Poutanen $M$, Perheentupa A, et al. Serum HE4 concentration is not dependent on menstrual cycle or hormonal treatment among endometriosis patients and healthy premenopausal women. Gynecol Oncol 2012;125:667-72.
52. Urban N, Thorpe JD, Bergan LA, Forrest RM, Kampani AV, Scholler $\mathrm{N}$, et al. Potential role of HE4 in multimodal screening for epithelial ovarian cancer. J Natl Cancer Inst 2011;103:1630-4.

53. Hess LM, Barakat R, Tian C, Ozols RF, Alberts DS. Weight change during chemotherapy as a potential prognostic factor for stage III epithelial ovarian carcinoma: a Gynecologic Oncology Group study. Gynecol Oncol 2007;107:260-5.

54. Ferraro S, Borille S, Caruso S, Boggio A, Muzio F, Rizzi A, et al. Body mass index does not influence human epididymis protein 4 concentrations in serum. Clin Chim Acta 2015;446:163-4.

55. Nagy B Jr, Krasznai ZT, Balla H, Csobán M, Antal-Szalmás P, Hernádi Z, et al. Elevated human epididymis protein 4 concentrations in chronic kidney disease. Ann Clin Biochem 2012;49:377-80.

56. Kappelmayer J, Antal-Szalmás P, Nagy B Jr. Human epididymis protein 4 (HE4) in laboratory medicine and an algorithm in renal disorders. Clin Chim Acta 2015;438:35-42.

57. Gizzo S, Ancona E, Saccardi C, D’Antona D, Nardelli GB, Plebani M. Could kidney glomerular filtration impairment represent the "Achilles heel" of HE4 serum marker? A possible further implication. Clin Chem Lab Med 2014;52:e45-6.

58. Lindquist JA, Mertens PR. Myofibroblasts, regeneration or renal fibrosis - is there a decisive hint? Nephrol Dial Transplant 2013;28:2678-81.

59. Lv YW, Yang L, Zhang M, Jiang LH, Niu JH, Hou J, et al. Increased human epididymis protein 4 in benign gynecological diseases complicated with chronic renal insufficiency patients. Genet Mol Res 2015;14:2156-61.

60. de Jonge MJ, Verweij J. Renal toxicities of chemotherapy. Semin Oncol 2006;33:68-73.

61. Ferraro S, Pasqualetti S, Carnevale A, Panteghini M. Cystatin C provides a better estimate of the effect of glomerular filtration rate on serum human epididymis protein 4 concentrations. Clin Chem Lab Med 2016;54:1629-34.

62. Macedo AC, da Rosa MI, Lumertz S, Medeiros LR. Accuracy of serum human epididymis protein 4 in ovarian cancer diagnosis: a systematic review and meta-analysis. Int J Gynecol Cancer 2014;24:1222-31.

63. Van Gorp T, Cadron I, Despierre E, Daemen A, Leunen K, Amant F, et al. HE4 and CA-125 as a diagnostic test in ovarian cancer: prospective validation of the risk of ovarian malignancy algorithm. Br J Cancer 2011;104:863-70.

64. Jacob F, Meier M, Caduff R, Goldstein D, Pochechueva T, Hacker N, et al. No benefit from combining HE4 and CA125 as ovarian tumor markers in a clinical setting. Gynecol Oncol 2011;121:487-91.

65. Moore RG, Miller MC, Disilvestro P, Landrum LM, Gajewski W, Ball JJ, et al. Evaluation of the diagnostic accuracy of the risk of ovarian malignancy algorithm in women with a pelvic mass. Obstet Gynecol 2011;118:280-8.

66. Lin J, Qin J, Sangvatanakul V. Human epididymis protein 4 for differential diagnosis between benign gynecologic disease and ovarian cancer: a systematic review and meta-analysis. Eur J Obstet Gynecol Reprod Biol 2013;167:81-5.

67. NCCN Clinical Practice Guidelines in Oncology, Ovarian Cancer (Including Fallopian Tube Cancer and Primary Peritoneal Cancer). Version 2.2018. https://www.nccn.org/ store/login/login.aspx?ReturnURL=https://www.nccn.org/ professionals/physician_gls/pdf/ovarian.pdf. Accessed date: October 2018. 
68. Ferraro S, Robbiano C, Tosca N, Panzeri A, Paganoni AM, Panteghini M. Serum human epididymis protein $4 \mathrm{vs}$. carbohydrate antigen 125 in ovarian cancer follow-up. Clin Biochem 2018;60:84-90.

69. Wang Y, Narla S, Champion-Lyons E, Graybill W, Zhu Y. Alteration of serum CA125 and HE4 levels in response to chemotherapy in patients with different histological subtypes of ovarian cancer, 69th AACC Annual Scientific Meeting Abstracts, 2017 (Abstract A-044).

70. Baumann KH, Wagner U, du Bois A. The changing landscape of therapeutic strategies for recurrent ovarian cancer. Future Oncol 2012;8:1135-47.

71. Rustin GJ, van der Burg ME, Griffin CL, Guthrie D, Lamont A, Jayson GC, et al. Early versus delayed treatment of relapsed ovarian cancer (MRC OV05/EORTC 55955): a randomised trial. Lancet 2010;376:1155-63.

72. Piovano E, Attamante L, Macchi C, Cavallero C, Romagnolo C, Maggino T, et al. The role of HE4 in ovarian cancer follow-up: a review. Int J Gynecol Cancer 2014;24:1359-65.

73. Scaletta G, Plotti F, Luvero D, Capriglione S, Montera R, Miranda A, et al. The role of novel biomarker HE4 in the diagnosis, prognosis and follow-up of ovarian cancer: a systematic review. Expert Rev Anticancer Ther 2017;17:827-39.
74. Piovano E, Cavallero C, Fuso L, Viora E, Ferrero A, Gregori G, et al. Diagnostic accuracy and cost-effectiveness of different strategies to triage women with adnexal masses: a prospective study. Ultrasound Obstet Gynecol 2017;50:395-403.

75. Barker PE. Cancer biomarker validation: standards and process: roles for the National Institute of Standards and Technology (NIST). Ann N Y Acad Sci 2003;983:142-50.

76. Schmitt M, Harbeck N, Daidone MG, Brynner N, Duffy MJ, Foekens JA, et al. Identification, validation, and clinical implementation of tumor-associated biomarkers to improve therapy concepts, survival, and quality of life of cancer patients: tasks of the Receptor and Biomarker Group of the European Organization for Research and Treatment of Cancer. Int J Oncol 2004;25: 1397-406.

77. Braicu El, Fotopoulou C, Van Gorp T, Richter R, Chekerov R, Hall C, et al. Preoperative HE4 expression in plasma predicts surgical outcome in primary ovarian cancer patients: results from the OVCAD study. Gynecol Oncol 2013;128:245-51.

78. Monaghan PJ, Lord SJ, St John A, Sandberg S, Cobbaert CM, Lennartz L, et al. Biomarker development targeting unmet clinical needs. Clin Chim Acta 2016;460:211-9. 